\title{
Anordnung gegen Massendatenabgleich zwischen WhatsApp und Facebook
}

Der Hamburgische Beauftragte für Datenschutz und Informationsfreiheit hat am 27. September 2016 eine Verwaltungsanordnung erlassen, die es Facebook ab sofort untersagt, Daten von deutschen WhatsApp-Nutzern zu erheben und zu speichern. Facebook wird ferner aufgegeben, bereits durch WhatsApp an das Unternehmen übermittelte Daten zu löschen.

Facebook und WhatsApp sind selbstständige Unternehmen, die die Daten ihrer jeweiligen Nutzer auf Grundlage ihrer eigenen Nutzungs- und Datenschutzbedingungen verarbeiten. Nach dem Erwerb von WhatsApp durch Facebook vor zwei Jahren haben sie öffentlich zugesichert, dass die Daten der Nutzer nicht miteinander ausgetauscht werden. Dass dies nun doch geschieht, ist nicht nur eine Irreführung der Nutzer und der Öffentlichkeit, sondern stellt auch einen Verstoß gegen das nationale Datenschutzrecht dar. Denn ein solcher Austausch ist nur dann zulässig, wenn sowohl auf Seiten des Unternehmens, das Daten liefert (WhatsApp) als auch bei dem empfangenden Unternehmen (Facebook) eine Rechtsgrundlage dafür vorliegt. Facebook hat allerdings weder eine wirksame Einwilligung von den Nutzern von WhatsApp eingeholt, noch ist eine gesetzliche Grundlage für den Datenempfang vorhanden. Dass Facebook die Regelungen des deutschen Datenschutzrechts respektieren muss, ist klar, nachdem im Juli der EuGH in einem Urteil bestätigt hat, dass nationales Datenschutzrecht anwendbar ist, wenn ein Unternehmen im Zusammenhang mit einer nationalen Niederlassung Daten verarbeitet. Dies tut Facebook in Deutschland durch seine Niederlassung in Hamburg, die das deutschsprachige Werbegeschäft betreibt.

\section{Hierzu der Hamburgische Beauftragte für Datenschutz und Informationsfreiheit, Johannes Caspar:}

„Die Anordnung schützt die Daten der ca. 35 Millionen WhatsApp-Nutzer in Deutschland. Es muss ihre jeweilige Entscheidung sein, ob sie eine Verbindung ihres Kontos mit Facebook wünschen. Dazu muss Facebook sie vorab um Erlaubnis fragen. Dies ist nicht geschehen.

Dazu kommen noch viele Millionen Personen, deren Kontaktdaten aus den Adressbüchern der Nutzer zu WhatsApp hochgeladen wurden, ohne dass diese etwas mit Facebook oder WhatsApp zu tun haben müssen. Diese gigantische Menge von Daten hat Facebook zwar nach eigenem Bekunden noch nicht erhoben.
Die Antwort von Facebook, dass dies lediglich zurzeit noch nicht erfolgt sei, gibt jedoch Anlass zur Sorge, dass das Ausmaß des Datenverstoßes noch massivere Auswirkungen nach sich ziehen wird."

\section{Das ist der Anlass für die Verwaltungsanordnung - WhatsApp Blog vom 25. August 2016 - Auszug \\ Die Zukunft für WhatsApp ${ }^{1}$}

Heute aktualisieren wir zum ersten Mal seit vier Jahren die Nutzungsbedingungen und Datenschutzrichtlinie von WhatsApp, als Teil unserer Pläne in den kommenden Monaten Wege zu testen, wie Personen mit Firmen kommunizieren können. Die aktualisierten Dokumente spiegeln auch wider, dass wir jetzt ein Teil von Facebook sind und wir kürzlich viele neue Funktionen, wie Ende-zu-Ende-Verschlüsselung, WhatsApp Call und MessagingWerkzeuge, wie WhatsApp für Web und Desktop eingeführt haben. Du kannst die vollständigen Dokumente hier lesen. Wir benachrichtigen alle, die die neusten unterstützten Versionen unserer App installiert haben, über diese Aktualisierungen und du wirst gebeten werden, auf „Zustimmen“ zu tippen, um den aktualisierten Bedingungen zuzustimmen.

Menschen verwenden unsere App jeden Tag, um mit Freunden und Familie in Kontakt zu bleiben und das ändert sich nicht. Wie wir Anfang des Jahres bekanntgegeben haben, möchten wir Wege erkunden, wie du mit Unternehmen, die dir wichtig sind, kommunizieren kannst, während wir dir weiterhin ein Erlebnis ohne Bannerwerbung von Dritten oder Spam bieten. Ob es eine Nachricht von deiner Bank ist, die dir eine möglicherweise betrügerische Transaktion meldet oder eine Nachricht einer Fluggesellschaft, die dich über eine Flugverspätung informiert - viele von uns bekommen Informationen auf verschiedenen Wegen, inklusive SMS oder über Anrufe. Wir möchten diese Funktionen in den nächsten Monaten testen, aber müssen dafür unsere Nutzungsbedingungen und Datenschutzrichtlinie ${ }^{2}$ aktualisieren.

\footnotetext{
1 https://blog.whatsapp.com/10000627/Die-Zukunft-f\%C3\%BCr-WhatsApp
}

2 https://www.whatsapp.com/legal/\#privacy-policy 